\title{
Diabetesmanifestation im Kindesalter: Alltagsbelastungen und berufliche Entwicklung der Eltern
}

B. Müller ${ }^{3}$

H.-P. Schwarz ${ }^{5}$

V. Hesse ${ }^{4}$

P. Busse-Widmann ${ }^{5}$

\section{Diabetes in childhood: burden and professional conse- quences for parents}

Hintergrund und Fragestellung: Welche Belastungen und beruflichen sowie finanziellen Folgen erleben Mütter und Väter nach der Diabetesmanifestation bei ihrem Kind abhängig vom Manifestationsalter und der familiären Situation?

Patienten und Methodik: An vier großen pädiatrischen Diabeteszentren erhielten die Familien aller dort behandelten Kinder, die eine Diabetesdauer $<10$ Jahren und ein Manifestationsalter $<14$ Jahren aufwiesen, einen halbstandardisierten Fragebogen (subjektive Belastung, Berufstätigkeit, Berufsplanung, finanzielle Folgen jeweils für beide Elternteile, demografische Daten).

Ergebnisse: An der Studie nahmen 580 Familien mit 583 Kindern mit Typ-1-Diabetes teil (46\% Mädchen, Diabetesdauer 5,0 \pm 3,2 Jahre, Manifestationsalter 6,9 \pm 3,9 Jahre). 42\% der Kinder erkrankten vor dem 6 . Lebensjahr. $11 \%$ der Eltern waren allein erziehend. Vor der Manifestation waren 93\% der Väter vollzeitberufstätig, danach änderten $4 \%$ ihre Tätigkeit. Die Mütter waren vor der Manifestation zu 22\% vollzeit- und zu 38\% teilzeitberufstätig; davon schränkten $31 \%$ die Tätigkeit ein oder gaben sie auf. Die berufliche Planung wurde bei 33\% aller Mütter durch den Diabetes beeinträchtigt, besonders betroffen waren davon mit 44\% diejenigen, deren Kind vor dem 6 . Lebensjahr erkrankte. Finanzielle Einschränkungen beschrieben $44 \%$ der Familien. Die Mehrbelastung im Alltag (Skala von 1 bis 5) war bei Müttern und Vätern umso höher, je jünger das Kind an Diabetes erkrankte. Die subjektive Belastung der Mütter $(3,6 \pm 1,1)$ war höher als die der Väter $(2,8 \pm 1,1)(p=0,001)$.

Folgerung: Es besteht akuter Handlungsbedarf, um die Belastungen der Familien zu reduzieren und die berufliche und soziale Integration der Mütter von jüngeren Kindern mit Diabetes durch Verbesserung außerfamiliärer Angebote zu ermöglichen.
Background and objective: To investigate the burden and the financial and professional consequences for mothers and fathers after the onset of diabetes in their child in relationship to age at onset and family structure.

Patients and methods: All families of children with an age at onset $<14$ years and a diabetes duration $<10$ years treated at four large pediatric diabetes centers received a structured questionnaire (burden of diabetes, professional position and career development, financial consequences for both parents, demographic data).

Results: 580 families with 583 children with type 1 diabetes (46\% girls, diabetes duration 5,0 $\pm 3,2$ years, age at onset $6.9 \pm$ 3.9 years) participated. $42 \%$ of the children had an age at onset below 6 years. $11 \%$ had single parents. Before the onset of diabetes in their children $93 \%$ of the fathers worked full-time, thereafter $4 \%$ changed their employment. Mothers worked at onset full-time in $22 \%$ and part-time in $38 \%$; thereafter $31 \%$ reduced their working time or stopped working. $33 \%$ of the mothers reported handicaps in their professional career development, especially those with a child with age at onset below 6 years (44\%). Negative financial consequences were present in $44 \%$ of the families. The day to day burden on a scale from 1 to 5 decreased both in mothers and in fathers with increasing age at onset. The individual burden was higher in mothers $(3,6 \pm 1,1)$ than in fathers $(2,8 \pm 1,1)(\mathrm{p}=0.000)$.

Conclusions: Initiatives to reduce the burden on families with a child with diabetes are urgently needed. Particularly the social and professional integration of mothers with younger children at diabetes onset need to be improved through support measures outside the family.

${ }^{1}$ Medizinische Hochschule Hannover

${ }^{2}$ Kinderkrankenhaus auf der Bult, Hannover

Klinik für Allgemeine Pädiatrie am Charité-Virchow-Klinikum, Berlin

${ }^{4}$ Krankenhaus Berlin-Lichtenberg, Klinik für Kinder- und Jugendmedizin Lindenhof

${ }^{5}$ Dr. von Haunersches Kinderspital, Ludwig-Maximilians-Universität München

Korrespondenz

Priv.-Doz. Dr. Karin Lange - Abteilung Medizinische Psychologie OE 5430

Medizinische Hochschule Hannover · Carl-Neuberg-Straße 1 · 30625 Hannover · Tel.: 0511/5324437

Fax: 0511/5324214 · E-Mail: Lange.Karin@mh-hannover.de

eingereicht: $16.2 .2004 \cdot$ alkzeptiert: 29.4 .2004 
Die intensivierte Insulintherapie und auch die Insulinpumpentherapie haben sich in den vergangenen 10 Jahren in Deutschland und auch international zur Therapie der Wahl für Kinder mit Typ-1-Diabetes entwickelt $(6,13,20)$. Damit wird von Manifestation an eine möglichst normnahe Stoffwechseleinstellung angestrebt, welche die Kinder vor akuten Komplikationen ebenso wie langfristig vor Folgeerkrankungen schützen soll (7). Darüber hinaus zählt die normale körperliche, seelische und soziale Entwicklung eines Kindes mit Typ-1-Diabetes, d. h. auch die schulische und soziale Integration, zu den zentralen Therapiezielen in der pädiatrischen Diabetologie $(3,14)$. Parallel zu dieser Entwicklung vollzog sich in der Bundesrepublik Deutschland in den vergangenen 10 Jahren ein deutlicher gesellschaftlicher Wandel, der vor allem durch eine steigende Zahl berufstätiger Elternpaare und allein Erziehender gekennzeichnet ist $(5,23)$. In einer Querschnittstudie an vier pädiatrischen Zentren wurde vor diesem Hintergrund untersucht, welche Folgen die Diabetesmanifestation bei Kindern mit Blick auf zeitgemäße Therapiekonzepte und aktuelle soziale Bedingungen für Familien hat. Dabei stehen die berufliche Entwicklung beider Eltern, die finanzielle Situation der Familien und die von Müttern und Vätern erlebte zusätzliche Belastung durch die Stoffwechselstörung im Vordergrund.

\section{Methodilk}

An vier großen deutschen pädiatrischen Diabeteszentren (DDG) (Kinderkrankenhaus auf der Bult Hannover, Universitätskinderklinik Berlin Charité, Dr. von Haunersches Kinderspital München, Kinderklinik Lindenhof Berlin-Lichtenberg) wurde Müttern und/oder Vätern, die während des dreimonatigen Studienzeitraums mit ihren Kindern die jeweilige Diabetesambulanz aufsuchten, ein halbstandardisierter Fragebogen ausgehändigt. Beide Elternteile bearbeiteten die Bögen zu Hause. Die Unterlagen konnten anonym an die Zentren geschickt oder dort jeweils in einen Sammelbehälter eingeworfen werden.

Einbezogen wurden alle Eltern, deren Kinder eine Diabetesdauer zwischen 3 Monaten und 10 Jahren und ein Manifestationsalter unter 14 Jahren aufwiesen. Der Fragebogen setzte sich aus 19 standardisierten Items und 6 offenen Fragen zusammen. Erhoben wurden demografische Daten des Kindes, Daten zur Familienstruktur (Kinderzahl, Zusammensetzung der Kernfamilie), das Ausbildungsniveau von Mutter und Vater, die Berufstätigkeit beider Eltern vor und nach der Diabetesmanifestation und mögliche Einflüsse des Diabetes auf die berufliche Entwicklung beider Eltern. Außerdem schätzten Mütter und Väter ihre subjektive zusätzliche Belastung seit der Erkrankung des Kindes im Vergleich zu einem stoffwechselgesunden Kind auf einer 5-stufigen Skala ein. Weiterhin wurde nach finanziellen Einbußen durch den Diabetes gefragt. Die sechs offenen Fragen boten Müttern und Vätern die Möglichkeit, berufliche oder ggf. andere Folgen des Diabetes detailliert zu schildern.

\section{Stichprobe}

Während der dreimonatigen Erhebungsphase gaben 580 Familien (mit 583 Kindern mit Typ-1-Diabetes) den Bogen zurück (Rücklauf Hannover $\mathrm{n}=224$ (93\%), Berlin Charité $\mathrm{n}=206$ (78\%), München $\mathrm{n}=103$ (77\%), Berlin-Lichtenberg $\mathrm{n}=47$ (88\%)). Der Rücklauf betrug damit $84 \%$ der in den Zentren ambulant behandelten Kinder und Jugendlichen, die den Einschlusskriterien entsprachen. Es antworteten 519 Elternpaare, 57 allein erziehende Mütter bzw. 4 Väter (letztere zusammen 10,5\%). Der Anteil allein erziehender Eltern war in den
Tab.1 Berufstätigkeit der Eltern zum Zeitpunkt der Diabetesmanifestation beim Kind.

Alter des Kindes bei <
Manifestation:

\begin{tabular}{|c|c|c|c|}
\hline \multicolumn{4}{|l|}{ Mutter: } \\
\hline Hausfrau/Erziehungszeit & $50,0 \%$ & $38,6 \%$ & $24,2 \%$ \\
\hline teilzeitberufstätig & $32,9 \%$ & $35,9 \%$ & $50,3 \%$ \\
\hline vollzeitberufstätig & $17,1 \%$ & $25,5 \%$ & $25,5 \%$ \\
\hline \multicolumn{4}{|l|}{ Vater: } \\
\hline keine Berufstätigkeit/Rente & $4,9 \%$ & $4,9 \%$ & $8,1 \%$ \\
\hline teilzeitberufstätig & $1,3 \%$ & $1,2 \%$ & $0,0 \%$ \\
\hline vollzeitberufstätig & $93,8 \%$ & $93,9 \%$ & $91,9 \%$ \\
\hline \multicolumn{4}{|l|}{ beide Eltern } \\
\hline berufstätig & $46,3 \%$ & $57,2 \%$ & $67,7 \%$ \\
\hline
\end{tabular}

Tab.2 Anteile von allen befragten Müttern (Berufstätige und Hausfrauen), die ihre Berufstätigkeit nach der Diabetesmanifestation bei ihrem Kind aufgaben, die Tätigkeit einschränkten oder die ihre beruflichen Zukunftspläne revidierten.

\begin{tabular}{llll}
\hline $\begin{array}{l}\text { Alter des Kindes bei } \\
\text { Manifestation: }\end{array}$ & $<6$ Jahre & $\mathbf{6}$ bis 10 Jahre & $\mathbf{1 1}$ bis 14 Jahre \\
\hline $\begin{array}{l}\text { berufliche Folgen für die } \\
\text { Mutter: }\end{array}$ & & & \\
\hline $\begin{array}{l}\text { Aufgabe der Berufstätigkeit } \\
\text { Änderung der Berufstätigkeit }\end{array}$ & $20,5 \%$ & $12,0 \%$ & $6,6 \%$ \\
\hline \begin{tabular}{l} 
Änderung der beruflichen Planung \\
\hline
\end{tabular} & $44,1 \%$ & $34,5 \%$ & $21,1 \%$ \\
\hline
\end{tabular}

Zentren unterschiedlich: Charité Berlin 15,1\%, Hannover 9,8\%, München 4,9\% und Berlin-Lichtenberg 4,3\%. Von den 583 Kindern und Jugendlichen waren 45,6\% weiblich. Das mittlere Alter bei Manifestation betrug 6,9 $\pm 3,9$ Jahre, das aktuelle Alter 11,9 \pm 3,9 Jahre und die Diabetesdauer entsprechend 5,0 \pm 3,2 Jahre. Zum Zeitpunkt der Manifestation waren 41,6\% der Kinder jünger als 6 Jahre alt, 32,0\% waren 6-10 Jahre alt und 26,4\% waren zwischen 11 und 14 Jahren alt. Die Stichproben der vier Zentren waren hinsichtlich dieser demografischen Parameter vergleichbar. Die familiäre Situation der 583 Kinder mit Typ-1-Diabetes war wie folgt charakterisiert: 21\% waren Einzelkinder, 51\% hatten ein Geschwister, 16\% zwei Geschwister und die verbleibenden $12 \%$ drei bis sieben Geschwister (mittlere Kinderzahl: 2,1 $\pm 1,01$ ). Die mittlere Kinderzahl von Elternpaaren $(2,1 \pm 1,02)$ unterschied sich nicht von der allein erziehender Eltern $(2,0 \pm 1,01)(\mathrm{p}=0,141)$.

\section{Statistik}

Die Daten wurden mit dem Programm SPSS 11.5 für Windows zentral ausgewertet und zunächst deskriptiv als Häufigkeitsverteilungen und Mittelwerte dargestellt. Die freien Antworten wurden vordefinierten Kategorien zugeordnet. Mittelwertsvergleiche erfolgten mittels Student-t-Tests, zweifaktorieller ANOVA oder dem Wilcoxon-Test bzw. dem Mann-Whitney-U-Test. Als Zusammenhangsmaße dienten Spearman's rho oder der Chi $^{2}$-Test. Als Signifikanzniveau wurde $\alpha=0,01$ gewählt. 
Tab.3 Veränderungen in der mütterlichen Berufstätigkeit in den vier beteiligten pädiatrischen Diabeteszentren (jeweils bezogen auf alle beteiligten Mütter).

\begin{tabular}{|c|c|c|c|c|c|}
\hline & $\begin{array}{l}\text { München } \\
n=103\end{array}$ & $\begin{array}{l}\text { Hannover } \\
n=224\end{array}$ & $\begin{array}{l}\text { Charité Berlin } \\
n=203\end{array}$ & $\begin{array}{l}\text { Berlin-Lichtenberg } \\
n=47\end{array}$ & $\begin{array}{l}\text { alle } \\
n=577\end{array}$ \\
\hline Aufgabe der Tätigkeit & $19,0 \%$ & $18,8 \%$ & $8,4 \%$ & $8,5 \%$ & $14 \%$ \\
\hline Änderung der Tätigkeit & $30,1 \%$ & $24,6 \%$ & $26,7 \%$ & $23,4 \%$ & $26 \%$ \\
\hline veränderte Planung & $45,1 \%$ & $38,4 \%$ & $27,7 \%$ & $21,7 \%$ & $33 \%$ \\
\hline
\end{tabular}

\section{Ergebnisse}

\section{Ausbildung und Berufstätigkeit der Eltern vor der Diabetes- manifestation}

Die höchsten Schulabschlüsse der Mütter/Väter waren wie folgt verteilt: ohne Abschluss (2/2\%), Hauptschule (20/23\%), Realschule (43/ $38 \%$ ) und Abitur (35/37\%). Das Bildungsniveau der Mütter und Väter war vergleichbar $(\mathrm{p}=0,559)$. Auch zwischen den Zentren ergaben sich hier keine Unterschiede (Mütter $p=0,288$; Väter $p=0,301$ ). Die Daten zur beruflichen Situation der Mütter zum Zeitpunkt der Manifestation (Tab.1) deckten sich mit den repräsentativen Angaben des Mikrozensus (MZ) 2001 (23), wonach in Deutschland 52,9\% der Mütter mit Kindern unter 6 Jahren und 62,4\% der Mütter mit Kindern unter 15 Jahren berufstätig sind.

Allein erziehende Mütter waren hier mit 75,1\% häufiger berufstätig als Mütter aus Zweielternfamilien mit 58,9\% ( $p=0,001)$. Weiterhin zeigte sich auch in dieser Stichprobe die im Mikrozensus beschriebene Tendenz der Mütter, mit zunehmender Kinderzahl die Berufstätigkeit aufzugeben. 67\% der Mütter mit einem Kind waren berufstätig (MZ: 66\%), 63\% der Mütter mit zwei Kindern (MZ: 65\%) und 48\% der Mütter mit drei und mehr Kindern (MZ: 47\%). Der Anteil vollzeitberufstätiger Frauen stieg mit dem Ausbildungsniveau an: ohne Abschluss 11,0\%, Hauptschule $11,9 \%$, Realschule 24,8\%, Abitur 25,8\% ( $p=0,001)$. Der Vergleich der vier Kinderkliniken zeigt einen relativ hohen Anteil vollzeitberufstätiger Mütter in Berlin-Lichtenberg (64,2\%) gegenüber Berlin-Charité (26,9\%) sowie Hannover (14,8\%) und München $(11,2 \%)(p=0,001)$.

\section{Berufstätigkeit der Eltern nach der Diabetesmanifestation}

Der Anteil von allen befragten Müttern, die nach der Diabetesmanifestation ihre berufliche Tätigkeit aufgegeben, den Umfang der Tätigkeit reduziert oder ihre berufliche Zukunftsplanung revidiert hatten (z. B. späterer Wiedereinstieg nach der Erziehungszeit, Abbruch des Studiums), war vom Alter des Kindes abhängig (Tab.2) (jeweils $\mathrm{p}=0,001$ ).

Eine Beziehung zum Ausbildungsniveau der Mütter zeigte sich dabei nicht ( $p=0,584)$. Allein Erziehende und Mütter aus Zweielternfamilien veränderten ihre Berufstätigkeit und ihre Zukunftspläne in vergleichbarem Ausmaß ( $p=0,198$ bzw. $p=0,547$ ). Die Zahl der Väter, die angaben, aufgrund der Diabeteserkrankung ihres Kindes die Berufstätigkeit verändert $(4,2 \%)$ oder beendet zu haben $(1,9 \%)$ war gering.

Die qualitativen Angaben der Mütter zur Veränderung der Berufstätigkeit betrafen: 1. eine Verringerung des Stundenkontingents, 2. den Verzicht auf Schichtdienst/Bereitschaftsdienste/flexible Arbeits-
Tab.4 Mittlere subjektive Mehrbelastung der Mütter und Väter abhängig vom Alter des Kindes bei der Diabetesmanifestation (Skala: 1 = keine bis 5 = sehr hohe Mehrbelastung).

\begin{tabular}{lllll|}
\hline $\begin{array}{l}\text { Alter des Kindes bei } \\
\text { Manifestation: }\end{array}$ & <6 Jahre & 6 bis 10 Jahre & 11 bis 14 Jahre & \\
\hline Mütter & $3,97 \pm 1,0$ & $3,62 \pm 1,1$ & $3,14 \pm 1,1$ & $\mathrm{p}=0,001$ \\
Väter & $3,07 \pm 1,1$ & $2,69 \pm 1,1$ & $2,42 \pm 1,1$ & $\mathrm{p}=0,001$ \\
\hline
\end{tabular}

zeiten und 3. den Verzicht auf berufliche Fortbildungen oder Aufstieg. Die qualitativen Daten zeigten außerdem, dass Hausfrauen ihre Arbeitszeiten (Organisation im Haushalt, Versorgung während Kindergarten und Grundschule, Betreuung der gesunden Geschwisterkinder) weitestgehend auf das Kind mit Diabetes abstimmen mussten. Übereinstimmend begründeten die Mütter den Rückzug aus dem Berufsleben damit, dass außerhalb der Kernfamilie keine angemessene und verläßliche Betreuung des Kindes mit Diabetes zu realisieren sei bzw. dass von Kindergärten und Grundschulen die ständige Erreichbarkeit der Mutter erwartet werde. Weitere 4\% der Mütter gaben in den freien Texten an, dass sie ihre berufliche Tätigkeit wegen Selbständigkeit (z. B. Landwirtschaft, Gastronomie) oder aus finanziellen Gründen nicht einschränken konnten, obwohl es die angemessene Betreuung ihres Kindes erforderte. Beim Vergleich der vier pädiatrischen Diabeteszentren ergaben sich regionale Unterschiede darin, in welchem Ausmaß die Mütter durch den Diabetes in ihrer beruflichen Entwicklung beeinträchtigt wurden (Aufgabe der Tätigkeit $p=0,002$; Änderung $p=0,004$; Planung $p=0,006$ ) (Tab.3). Die im Vergleich mit den anderen Zentren relativ geringen beruflichen Einschränkungen der Mütter aus Berlin-Lichtenberg sind umso bemerkenswerter, als dort zum Zeitpunkt der Manifestation der höchste Anteil der Mütter berufstätig war.

\section{Erlebte Mehrbelastung im Vergleich zu einem gesunden Kind}

Mütter und Väter schätzten die eigene Mehrbelastung durch den Diabetes im Vergleich zu einem stoffwechselgesunden Kind auf einer fünfstufigen Skala von 1 (keine Mehrbelastung) bis 5 (sehr große Mehrbelastung) ein. Sie bezogen sich dabei im Rückblick auf den Zeitraum seit der Manifestation (Tab.4).

Die Belastung durch den Diabetes im Alltag korrelierte bei den Müttern $($ rho $=-, 32)$ und auch den Vätern $($ rho $=-, 27)$ negativ mit dem Alter des Kindes bei Manifestation $(p=0,001)$. Mütter waren dabei stärker belastet als die Väter. (3,6 $\pm 1,1$ vs. $2,8 \pm 1,1, p=0,001)$. Die Beziehung zur aktuellen Diabetesdauer war bei Müttern $($ rho $=0,080)$ und Vätern $($ rho $=0,032)$ schwach ausgeprägt. Nach Adjustierung hinsichtlich des Manifestationsalters unterschied sich die Belastung von allein erziehende Müttern nicht von der, die Mütter aus Zweielternfamilien angaben (3,52 $\pm 1,1$ vs. 3,61 $\pm 1,1 ; p=0,135)$. 
Außerdem zeigte sich, dass sich Hausfrauen $(3,9 \pm 1,1)$ stärker belastet erlebten als berufstätige Mütter $(3,4 \pm 1,1)(p=0,003)$. Zentrumseffekte ergaben sich weder für die Belastung der Mütter $(p=0,242)$ noch für die der Väter $(p=0,138)$.

\section{Finanzielle Folgen der Diabetesmanifestation für Familien}

Über finanzielle Einbußen seit der Diabetesmanifestation berichteten $43,8 \%$ der Familien. Dabei deutet sich wiederum eine Beziehung zum Manifestationsalter an (<6 Jahre: 46,9\%; 6-10 Jahre: 41,1\%; 11 bis 14 Jahre: $41,1 \%)$, die jedoch nicht signifikant war $(\mathrm{p}=0,454)$.

\section{Diskussion}

Parallel zur Entwicklung einer immer intensiveren Diabetestherapie auch in der Pädiatrie (13) vollzog sich in der Bundesrepublik Deutschland in den vergangenen 10 Jahren ein deutlicher gesellschaftlicher Wandel (5). Die vorliegende Arbeit beschreibt erstmals, welche Auswirkungen sich durch den medizinischen und gesellschaftspolitischen Wandel für Familien ergeben, wenn ein Kind an Diabetes erkrankt. Durch die chronische Krankheit kam es für die Familien nicht nur zu erheblichen zusätzlichen Anforderungen im Alltag mit hoher psychischer Belastung, sondern auch zu finanziellen Einbußen und deutlich eingeschränkten beruflichen Zukunftsperspektiven für viele Mütter.

Da Kinder bis ins frühe Jugendalter mit der eigenverantwortlichen Diabetesbehandlung intellektuell überfordert sind $(2,27)$, übernahmen die Mütter die Verantwortung für die intensivierte Insulintherapie. Insbesondere Klein- und Vorschulkinder waren dabei wegen mangelnder Krankheitseinsicht und fehlendem Verständnis für akute Komplikationen, vor allem für Hypoglykämien (18), auf eine ständige kompetente Überwachung angewiesen $(11,24)$. Auch im Grundschulalter sind Kinder trotz manueller Geschicklichkeit mit der abstrakten Aufgabe Diabetestherapie überfordert (17,26,27). Auch sie sind regelmäßig auf die fachkundige Unterstützung durch Erwachsene angewiesen (1,3), während sie sich gleichzeitig intellektuell normal entwickeln und altersentsprechende Schulleistungen erbringen $(19,21)$.

Die enge Verknüpfung von mehr oder weniger erfolgreicher Diabetestherapie und den sich wandelnden psychosozialen Bedingungen wird mit Blick auf den Hilfebedarf der betroffenen Kinder deutlich. Seit 1991 verdoppelte sich in Deutschland der Anteil der Kinder und Jugendlichen, die bei einem allein erziehenden Elternteil leben, im Jahr 2001 waren es 19\% der Minderjährigen (23). Weiterhin wird ein Trend zu Zweiverdienerfamilien verzeichnet, so dass derzeit in 63\% der deutschen Familien mit minderjährigen Kindern beide Eltern (teilzeit- und vollzeit-) berufstätig sind. In 26\% der Familien sind beide Eltern vollzeitberufstätig (23). Wie zu erwarten, spiegelt sich dieser gesellschaftliche Wandel von der Versorgerehe zur Zweiverdienerfamilie (5) auch in der Situation der hier einbezogenen 580 Familien wider, deren Kind an Diabetes erkrankte. Die Anforderungen einer modernen Diabetestherapie und die konkreten Bedingungen der außerfamiliären Betreuung machten es für diese Familien erforderlich, dass insgesamt 33\% der befragten Mütter ihre berufliche Planung revidieren mussten. Besonders häufig betroffen waren Mütter (44\%), deren Kinder vor dem Schulalter an Diabetes erkrankten. Obwohl die hier einbezogene Stichprobe nicht repräsentativ für die Bundesrepublik sein kann, zeichnen sich im Vergleich der Zentren aus den alten Bundesländern und dem Zentrum, deren Patienten vor allem aus dem Ostteil Berlins stammen, regionale Unterschiede in den beruflichen Folgen für die Mütter ab. Es kann spekuliert werden, ob durch den deutlich höheren Versorgungsgrad an Ganztagesbetreuung für Kindergarten- und Grundschulkinder (66\%) in den neuen Bundesländern gegenüber den alten Bundesländern (11\%) $(5,23)$ auch die regionalen Unterschiede in den Diabetesfolgen für Familien erklärt werden können. Eine repräsentative Studie unter Beteiligung pädiatrischer Diabeteszentren aus allen Bundesländern könnte hier nicht nur stabile Daten zu den beruflichen und ökonomischen Folgen des Diabetes für Familien liefern, sondern auch darüber, ob und wie außerfamiliäre Betreuung die Qualität der Stoffwechseleinstellung von Kindern mit Diabetes beeinflusst.

Nach Angabe der Mütter und Väter in dieser Umfrage wird die derzeit unzureichende Betreuungssituation von Kindern mit Typ-1-Diabetes auch dadurch verstärkt, dass die von den Kultus- und Sozialministerien der Länder publizierten Empfehlungen zur Betreuung dieser Kinder in öffentlichen Einrichtungen (auch in der Regelschule) mehrheitlich auf dem medizinischen Stand der 80er Jahre mit starren Therapieschemata und wenigen Injektionen täglich sind. Sie sehen keine verlässliche Unterstützung bei der modernen medikamentösen Therapie durch Lehrer oder Erzieher vor, z. B. der entsprechende Erlass des niedersächsischen Kultusministeriums vom 23.11.1988. Die Medikamentengabe ist zwar nach schriftlicher Absprache mit den Eltern möglich, jedoch zählen medizinisch indizierte Maßnahmen, d. h. Injektionen, nicht dazu. Im positiven Fall ist die Hilfe bei der Insulintherapie dem persönlichen - rechtlich unzureichend abgesicherten - Engagement der Erzieher und Lehrer zu verdanken. Der hohe Stellenwert der öffentlichen Erziehung, der im Elften Kinder- und Jugendbericht (5) unter dem Leitmotto „Aufwachsen in öffentlicher Verantwortung“ vermittelt wird, erfährt durch die derzeit unbefriedigenden Regelungen zur Unterstützung von Kindern mit Typ-1-Diabetes kaum konkrete Umsetzung. Eine länderübergreifende Aktualisierung der verschiedenen Erlasse der Sozial- und Kultusminister wäre ein erster Schritt. Das Statement der Amerikanischen Diabetes Gesellschaft zur Versorgung von Kindern mit Diabetes in Schulen und Tageseinrichtungen (1) könnte dabei als Orientierung dienen. Kernstück des amerikanischen Modells ist ein strukturierter Hilfeplan, der individuell für jedes Kind zwischen Eltern und Lehrern/Erziehern unter Einbeziehung des Diabetesteams fixiert wird und Pflichten sowie Rechte aller Beteiligten beschreibt. Im Sinne eines Case Managements, das zu den Aufgaben eines pädiatrischen Diabetesteams zählt und entsprechend von den Kostenträgern finanziert wird, könnten Familien entlastet und die Sicherheit von Kindern und außerfamiliären Betreuern verbessert werden.

Nahezu die Hälfte der befragten Eltern berichtete in dieser Studie von finanziellen Einbußen durch die chronische Krankheit ihres Kindes. Die gegenwärtigen konkreten Maßnahmen zur Reduktion krankheitsbedingter psychosozialer und finanzieller Belastungen für diese Familien sind in Deutschland sehr begrenzt. Ein finanzieller Nachteilsausgleich ist zwar über die kritisch bewertete Anerkennung des Kindes als „schwerbehindert“ mit dem Merkzeichen „H“ möglich (§ 69 SGB IX in Verbindung mit §48 SGB X). Der Ausgleich erfolgt jedoch über einen steuerfreien Pauschbetrag ( $\$ 33$ b EStG), der für Familien mit niedrigem Einkommen, z. B. wegen Aufgabe der Berufstätigkeit der Mutter, nahezu ohne Bedeutung ist. Die Anerkennung der Betreuung eines Kindes mit Diabetes im Rahmen der 
Pflegeversicherung (nach SGB XI) wurde durch das Urteil des Bundessozialgerichts vom 17.06.99/Az.: B 3 P 10/98 R abgewiesen. Nur in wenigen Ausnahmen wird Müttern heute noch ein Pflegegeld der Pflegestufe I zuerkannt, wie es von der AGPD (1997) (4) empfohlen wurde. Hier bestünde jedoch die Möglichkeit, Familien aus unteren sozioökonomischen Schichten, darunter viele allein Erziehende (5), unabhängig von deren Steuerpflicht zu unterstützen und deren Armutsrisiko zu verringern.

Die Bedeutung einer kompetenten Betreuung von Kindern mit Typ1-Diabetes unterstreichen diverse Studien, die zeigen, dass vor allem psychosoziale Faktoren, z. B. niedriger sozioökonomischer Status der Familie, Einelternfamilie (25), Zugehörigkeit zu einer Minorität (12), Überforderung der Eltern oder psychische Erkrankung der Mutter (im Überblick: 8, 16) in Beziehung zu häufigen akuten Komplikationen, stationären Aufnahmen und zu einer unbefriedigenden Stoffwechseleinstellung stehen. Die Prognose der unzureichend betreuten Kinder ist nicht nur aus somatischer Sicht $(9,22)$, sondern auch psychosozial ungünstig (8-10).

Es wäre zu untersuchen, ob mit sozialpolitischen Maßnahmen die Qualität der Stoffwechseleinstellung von Kindern mit Typ-1-Diabetes allgemein, insbesondere aber von Kindern aus Risikokonstellationen verbessert werden kann. Diese Maßnahmen betreffen eine bundeseinheitliche verbindliche Regelung zur Unterstützung der Kinder bei der intensivierten Insulintherapie in Kindergarten, Schule und gegebenenfalls Hort. Weiterhin könnte ein von der Steuerpflicht unabhängiger Nachteilsausgleich besonders für Mütter, deren Kind sehr jung an Diabetes erkrankt, diskutiert werden. Es bleibt zu prüfen, ob durch diese Maßnahmen familiäre Ressourcen für die adäquate medizinische und psychosoziale Betreuung von Kindern mit Diabetes im Alltag freigesetzt und damit deren langfristige Prognose verbessert werden können.

Autorenerklärung: Die Autoren erklären, dass sie keine finanziellen Verbindungen mit einer Firma haben, deren Produkt in diesem Artikel eine wichtige Rolle spielt (oder mit einer Firma, die ein Konkurrenzprodukt vertreibt).

\section{Literatur}

1 American Diabetes Association. Care of children with diabetes in the school and day care setting. Diabetes Care 2003; 26: S131-S135(Suppl 1

2 Anderson BJ, Auslander WF, Jung KC, Miller JP, Santiago JV. Assessing family sharing of diabetes responsibilities. J Pediatr Psychol 1990; 15: 477-492

3 Hürter P, Burger W, Schober E, Holl R, Klinghammer A AGPD Arbeitsgemeinschaft Pädiatrische Diabetologie. Qualitätssicherung in der Pädiatrischen Diabetologie. Monatsschrift für Kinderheilkunde 1995; 143: 1146-1149

${ }^{4}$ Borkenstein M, Burger W, Holl R, Lang E, Nietzschmann U AGPD Arbeitsgemeinschaft Pädiatrische Diabetologie. Stellungnahme der Arbeitsgemeinschaft für Pädiatrische Diabetologie zur Frage einer Gewährung von Pflegegeld nach SGB XI an Kinder mit Diabetes mellitus. Diabetologie Informationen 1997; 19: 265-267

${ }^{5}$ Bundesministerium FSFJ (Familie, Senioren, Frauen und Jugend). Elfter Kinder- und Jugendbericht. Bericht über die Lebenssituation junger Menschen und die Leistungen der Kinder- und Jugendhilfe in Deutschland. www.bmfsfj.de, 2002

${ }^{6}$ Danne T, Mortensen HB, Hougaard P et al. Persistent differences among centers over 3 years in glycemic control and hypoglycemia in a study of 3,805 children and adolescents with type 1 diabetes from the Hvidore Study Group. Diabetes Care 2001; 24: 1342-1347
7 DCCT Diabetes Control and Complications Trial Research Group. Effect of intensive diabetes treatment on the development and progression of long-term complications in adolescents with insulin-dependent diabetes mellitus: Diabetes Control and Complications Trial. J Pediatr 1994; 125: 177-188

8 Delamater AM, Jacobson AM, Anderson B et al. Psychosocial therapies in diabetes. Report of the Psychosocial Therapies Working Group. Diabetes Care 2001; 24: 1286-1292

9 DIABAUD2 . Factors influencing glycemic control in young people with type 1 diabetes in Scotland: a population-based study (DIABAUD2). Diabetes Care 2001; 24: 239-244

10 DiLiberti JH, Lorenz RA. Long-term trends in childhood diabetes mortality: 1968-1998. Diabetes Care 2001; 24: 1348-1352

11 Hatton DL, Canam C, Thorne S, Hughes AM. Parentś perception of caring an infant or toddler with diabetes. J Adv Nurs 1995; 22: 569-577

${ }^{12}$ Hecker W, Bartus B, Heinze E, Holl RW. Stoffwechseleinstellung des Diabetes mellitus Typ 1 bei Kindern und Jugendlichen deutscher und ausländischer Herkunft. Diab Stoffw 1998; 5: 177-180

13 Holl RW, Grabert M, Beyer P et al. Welche Insulinsubstitution führen Kinder und Jugendliche mit Typ-1-Diabetes in Deutschland heute durch? Analyse der DPV-Wiss-Daten 1995-2001. Diabetes und Stoffwechsel 2002; 11: 209-216

${ }^{14}$ ISPAD International society for pediatric and adolescent diabetes. Consensus guidelines for the management of type 1 diabetes mellitus in children and adolescents. www.ispad.org, 2000

15 Jarosz-Chobot P, Guthrie DW, Otto-Buczkowska E, Koehler B. Selfcare of young diabetics in practice. Med Sci Monit 2000; 6: 129-132

${ }^{16}$ Kovacs M, Charron-Prochownik D, Obrosky DS. A longitudinal study of biomedical and psychosocial predictors of multiple hospitalizations among young people with insulin-dependent diabetes mellitus. Diabet Med 1995; 12: 142-148

${ }^{17}$ Lange K, Kinderling S, Hürter P. Eine multizentrische Studie zur Prozeß- und Ergebnisqualität eines strukturierten Schulungsprogramms. Diab Stoffw 2001; 10: 59-65

18 Marrero DG, Guare JC, Vandagriff JL, Fineberg NS. Fear of hypoglycemia in the parents of children and adolescents with diabetes: maladaptive or healthy response? Diabetes Educ 1997; 23: 281-286

19 McCarthy AM, Lindgren S, Mengeling MA, Tsalikian E, Engvall J. Effects of diabetes on learning in children. Pediatrics 2002; 109: e9

20 Mortensen HB, Robertson KJ, Aanstoot HJ et al. Hvidore Study Group on Childhood Diabetes. Insulin management and metabolic control of type 1 diabetes mellitus in childhood and adolescence in 18 countries. Diabet Med 1998; 15: 752-759

${ }^{21}$ Nordham EA, Anderson PJ, Jacobs R, Hughes M, Warne GI, Werther GA. Neuropsychological profiles of children with type 1 diabetes 6 years after disease onset. Diabetes Care 2001; 24: 1541-1546

22 Rewers A, Chase HP, Mackenzie T et al. Predictors of acute complications in children with type 1 diabetes. JAMA 2002; 287: 2511-2518

${ }^{23}$ Statistisches Bundesamt. Statistisches Jahrbuch 2002 für die Bundesrepublik Deutschland. Stuttgart: Metzler-Poeschel, 2002

${ }^{24}$ Sullivan-Bolyai S, Deatrick J, Gruppuso P, Tamborlane W, Grey M. Constant vigilance: mother's work parenting young children with type 1 diabetes. J Pediatr Nurs 2003; 18: 21-29

25 Thompson SJ, Auslander WF, White NH. Comparison of single-mother and two-parent families on metabolic control of children with diabetes. Diabetes Care 2001; 24: 234-238

${ }^{26}$ Weissberg-Benchell J, Glasgow AM, Tynan WD, Wirtz P, Turek J, Ward J. Adolescent diabetes management and mismanagement. Diabetes Care 1995; 18: 77-82

27 Wysocki T, Taylor A, Hough BS, Linscheid TR, Yeates KO, Naglieri JA. Deviation from developmentally appropriate self-care autonomy. Association with diabetes outcomes. Diabetes Care 1997; 19: 121-125 